\title{
Comparison Study of Clinical 3D MRI Brain Segmentation Evaluation
}

\author{
Ting Song ${ }^{1}$, Elsa D. Angelini ${ }^{2}$, \\ Brett D. Mensh ${ }^{3}$, Andrew Laine ${ }^{1}$
}

\author{
1Heffiner Biomedical Imaging Laboratory \\ Department of Biomedical Engineering, \\ Columbia University, NY, USA \\ ${ }^{2}$ Ecole Nationale Supérieure des Télécommunications \\ Paris, France \\ ${ }^{3}$ Department of Biological Psychiatry, Columbia University, \\ College of Physicians and Surgeons, NY, USA
}




\section{Overview}

- Introduction

- Segmentation Methods

- Histogram Thresholding.

- Multi-phase Level Set.

- Fuzzy Connectedness.

- Hidden Markov Random Field Model and the Expectation-Maximization (HMRF-EM).

- Results \& Comparison of Methods

- Conclusions 


\section{Introduction}

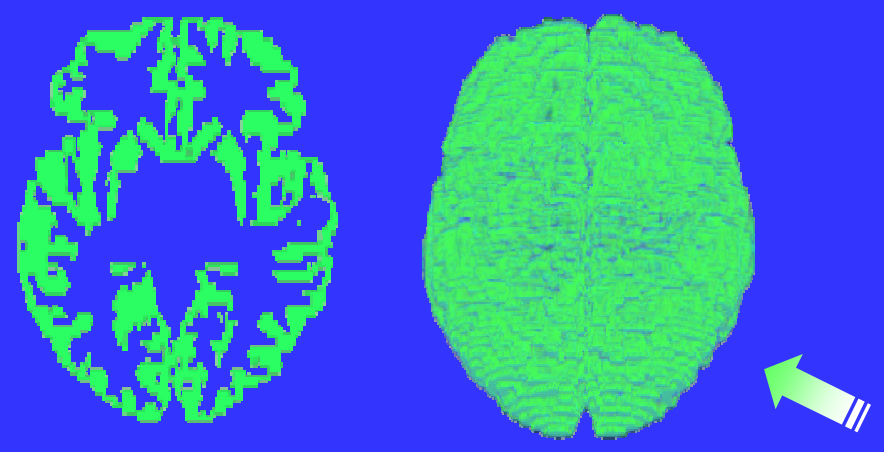

Gray Matter (GM)
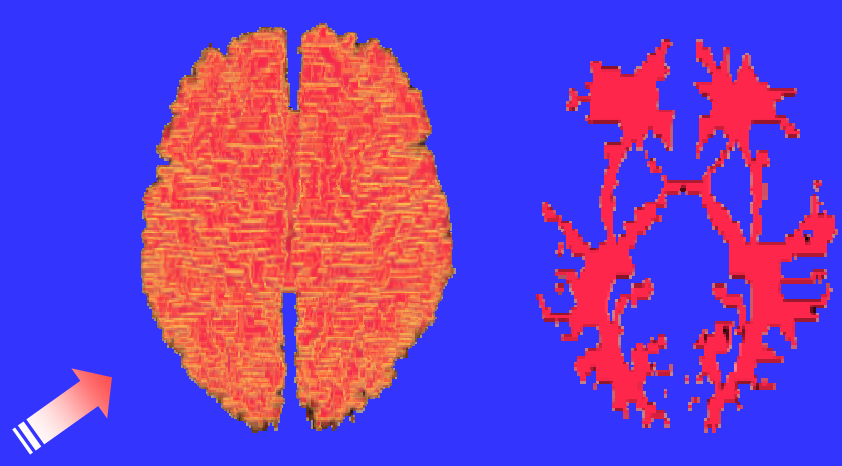

Whijte Maitter (WM)
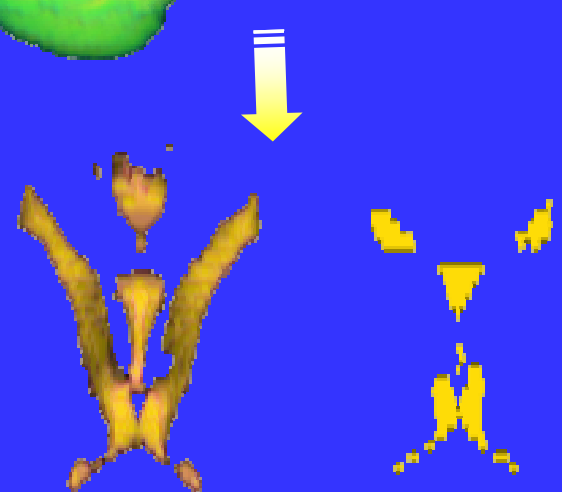

Cerebro-Spinal Fluid (CSF) 


\section{Motivation}

- Segmentation of clinical brain MRI data is critical for functional and anatomical studies of cortical structures.

- Little work has been done to evaluate and compare the performance of different segmentation methods on clinical data sets, especially for the CSF.

- The performance of four different methods was quantitatively assessed according to manually labeled data sets ("ground truth"). 


\section{Motivation}

Homogeneity of cortical tissues on simulated MRI data. (source: BrainWeb simulated brain database,

www.bic.mni.mcgill.ca/brainweb)

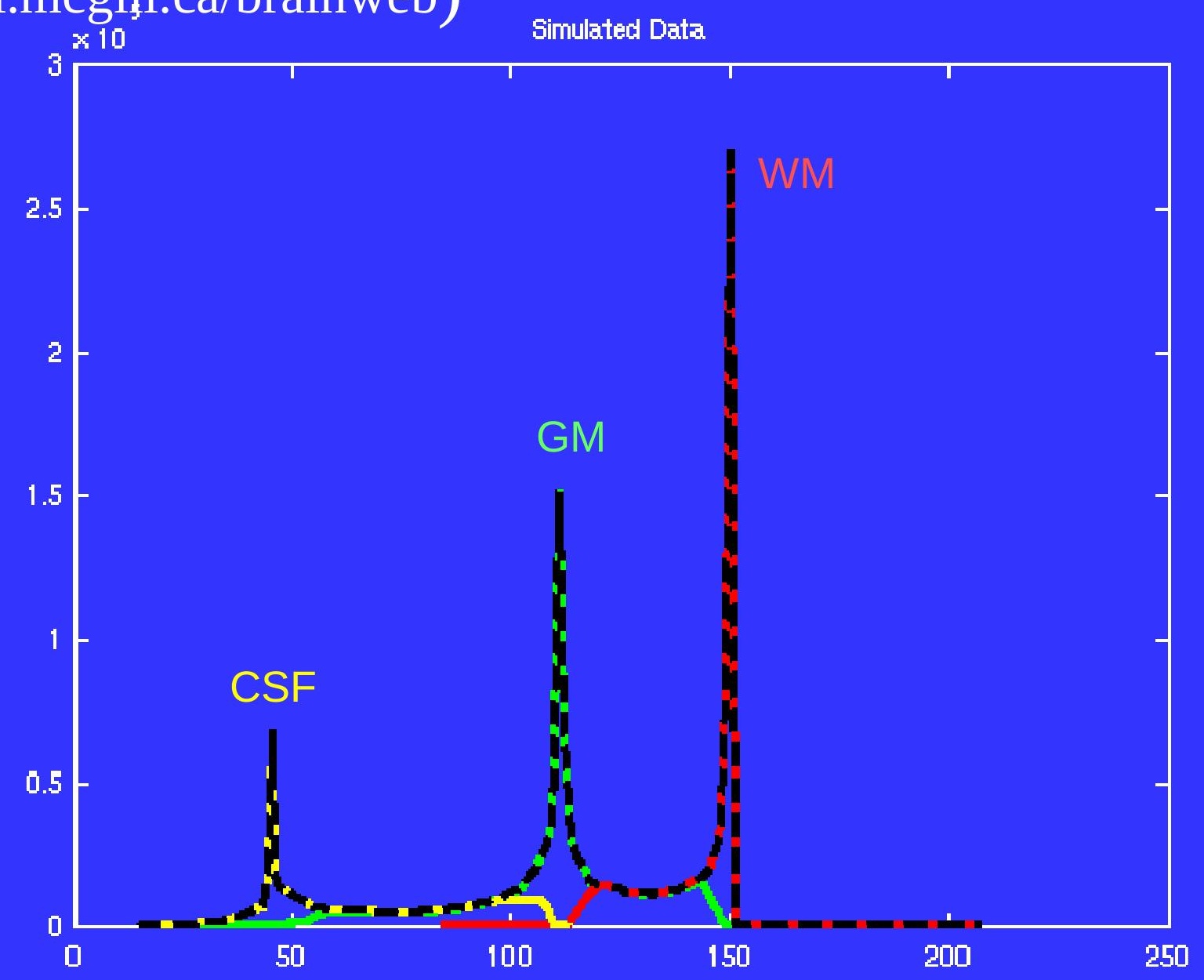




\section{Motivation}

Homogeneity of cortical tissues on clinical T1-weighted MRI data.

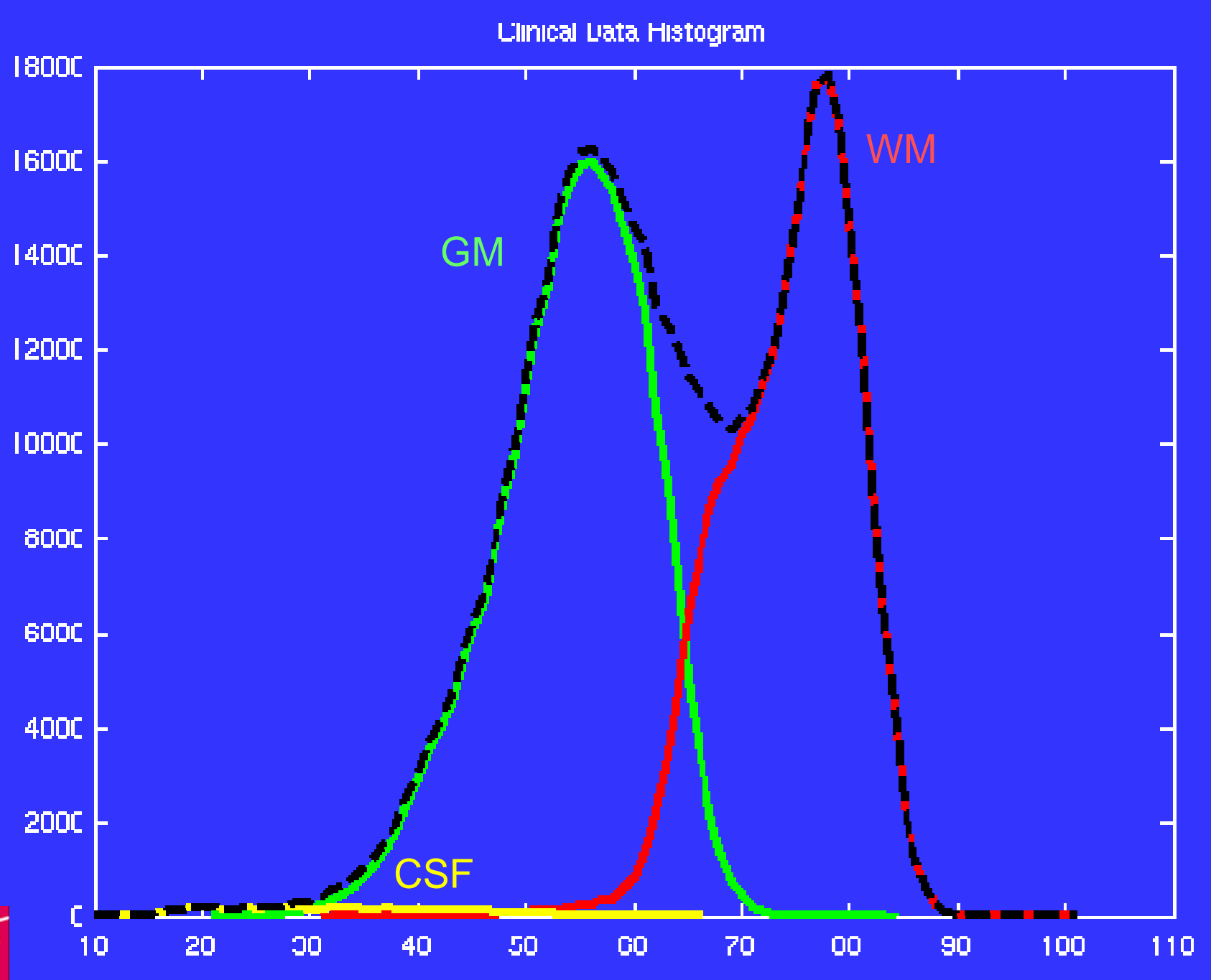




\section{Methodology}

Methods evaluated:

1. Histogram thresholding (Method A)

2. Multi-phase level set (Method B)

3. Fuzzy connectedness (Method C)

4. Hidden Markov Random Field Model and Expectation-Maximization (HMRF-EM) (Method D) 


\section{Histogram Thresholding}

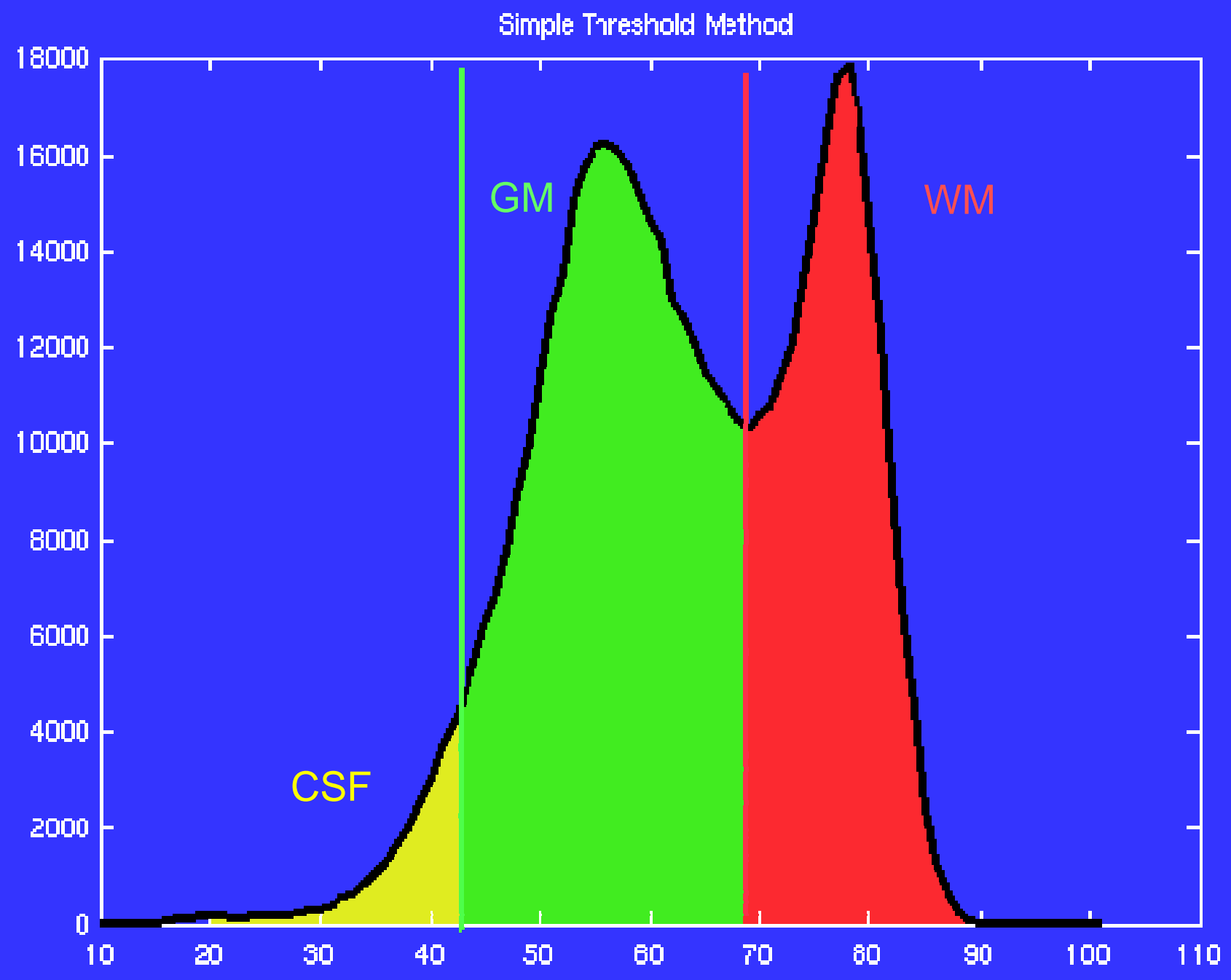




\section{Histogram Thresholding}

- Characteristics:

- Initialization with two threshold values.

- Simple set up \& fast computation.

- Set up for "optimal" performance:

- Tuning of threshold values for maximization of the Tanimoto index ( $\mathrm{TI})$ for the three tissues.

- Manually labeled data used as the reference.

- Simplex optimization for co-segmentation of the three tissues.

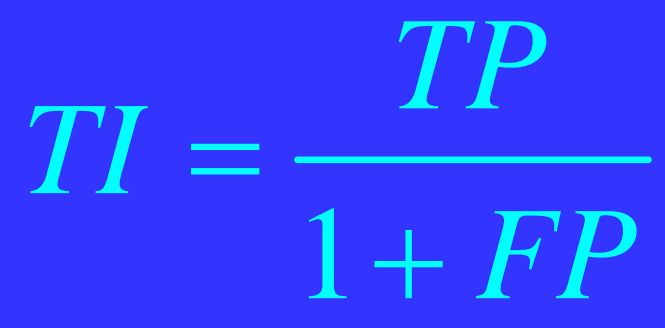




\section{Multi-Phase Level Set}

‘Active Contours Without Edges' [Chan-Vese IEEE TMI 2001]

- Method:

- 3D deformable model based on Mumford-Shah functional.

- Homogeneity-based external forces.

- Multiphase framework with 2 level set functions to segment 4 homogeneous objects simultaneously.

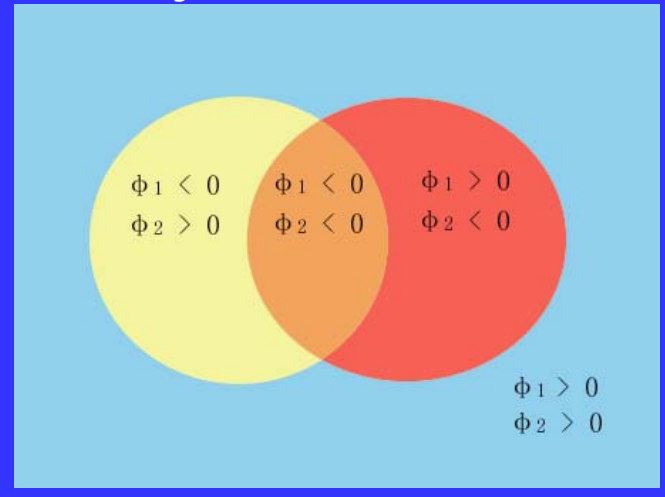
One $\phi$ function
$\Rightarrow$ Two phases
Two $\phi$ functions
$\Rightarrow>$ Four phases 


\section{Multi-Phase Level Set}

- Characteristics:

- Automatic initialization.

- No a priori information required.

- Set up:

- Details provided in:

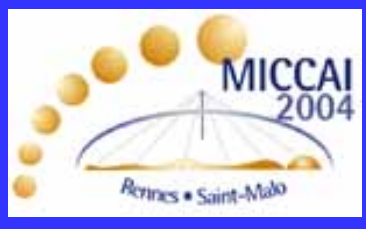

E. D. Angelini, T. Song, B. D. Mensh, A. Laine, "Multiphase three-dimensional level set segmentation of brain MRI," International Conference on Medical Image Computing and Computer-Assisted Intervention (MICCAI), Saint-Malo, France, September 2004. 


\section{Simple Fuzzzy Connectediness}

'Fuzzy Connectedness and Object Definition: Theory, Algorithms, and Applications in Image Segmentation', J. Udupa et al., GMIP, 1996.

- Method:

- Computation of a fuzzy connectedness map to measure similarities between voxels.

Affinity

High affinity

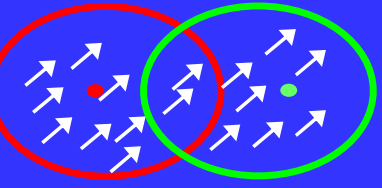

Low Affinity

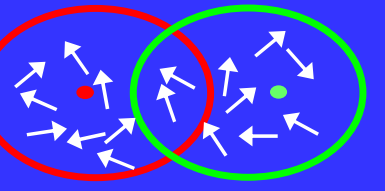

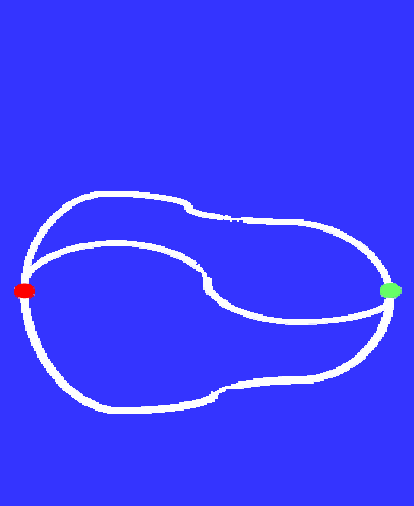

Connectedness
Fuzzy maps

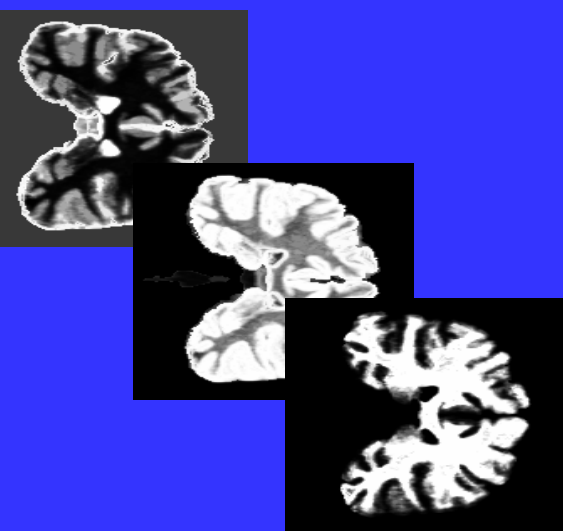

- Thresholding of each tissue fuzzy map to obtain a final segmentation. 


\section{Simple Fuzzy Connectedness}

- Characteristics:

- Initialization with seed points and prior statistics.

- Implementation from the National Library of Medicine Insight Segmentation and Registration Toolkit (ITK).

$$
\text { (www.itk.org) }
$$

- Set up for "optimal" performance:

- The threshold value for fuzzy maps was optimized using the Simplex scheme to obtain the segmentation with best accuracy (from the computed fuzzy connectedness map). 


\section{HMRF-EM}

'Segmentation of Brain MR Images Through a Hidden Markov Random Field Model and the Expectation-Maximization Algorithm '

[Y. Zhang, M. Brady, S. Smith, IEEE Transactions on Medical Imaging, 2001]

- Method

- Statistical classification method based on Hidden Markov random field models.

- Class labels, tissue parameters and bias fields are updated iteratively.

- Characteristics:

- The method was implemented in the FSL-FMRIB Software Library (http://www.fmrib.ox.ac.uk/fsl). 


\section{Results}

- Data

- Ten T1-weighted MRI data sets from healthy young volunteers.

- Data sets size $=(256 \times 256 \times 73)$ with $3 \mathrm{~mm}$ slice thickness and $0.86 \mathrm{~mm}$ in-plane resolution.

- Manual labeling available (manual protocol requiring 40 hours per brain). 


\section{Results}

- Evaluation protocol

- Measurements of organ's volume.

- True positive, false positive voxel fractions and the Tanimoto index for the each tissue.

- Analysis of variance (ANOVA) performed to evaluate the differences between the four segmentation methods. 


\section{Results}

\section{Segmentation of CSF}
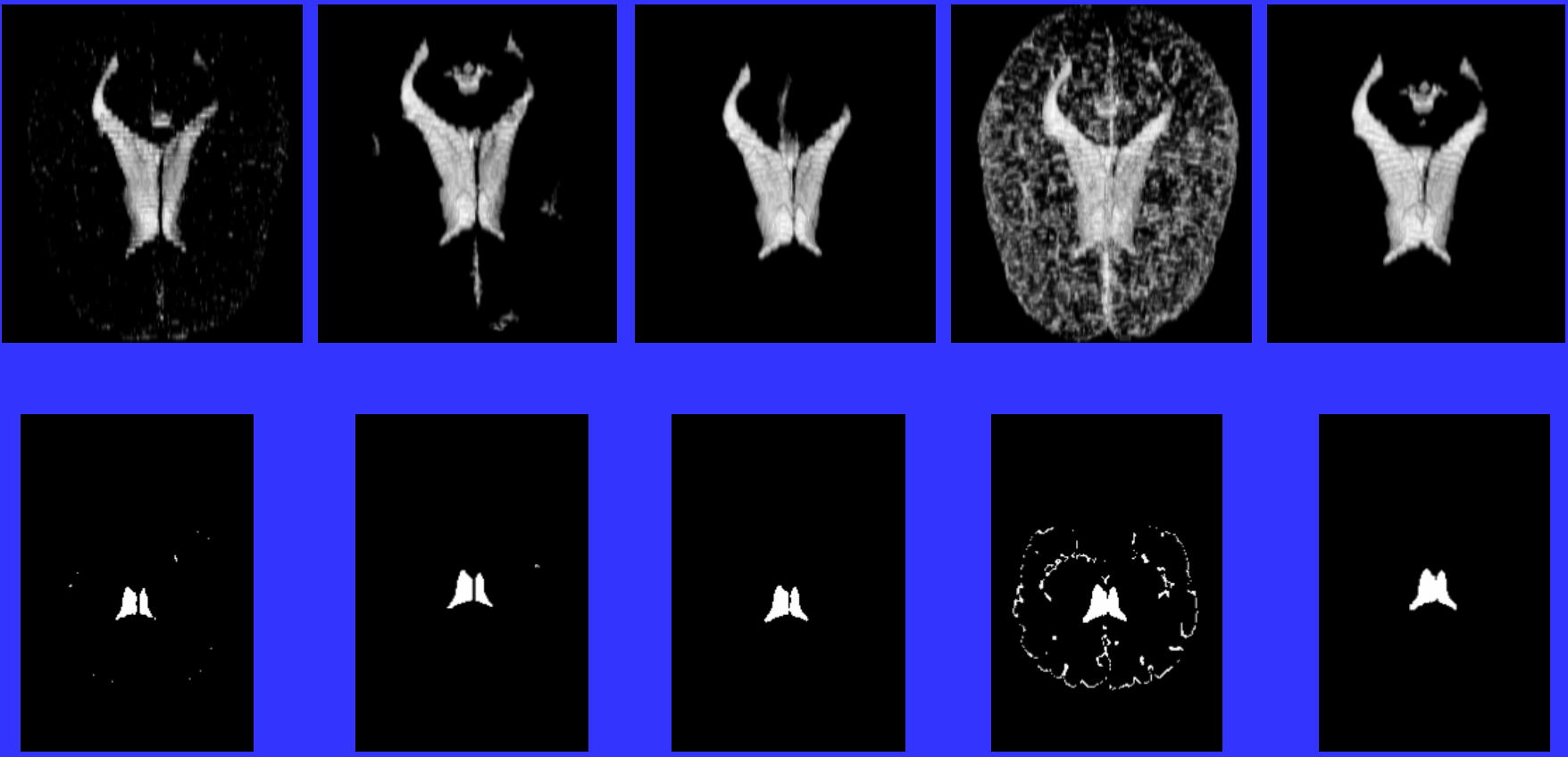

(a)

(b)

(c)

(d)

(e)

(a) Histogram thresholding, (b) Level set, (c) Fuzzy connectedness, (d) HMRFs, (e) Manual labeling. 


\section{Results}

\section{GM volume}

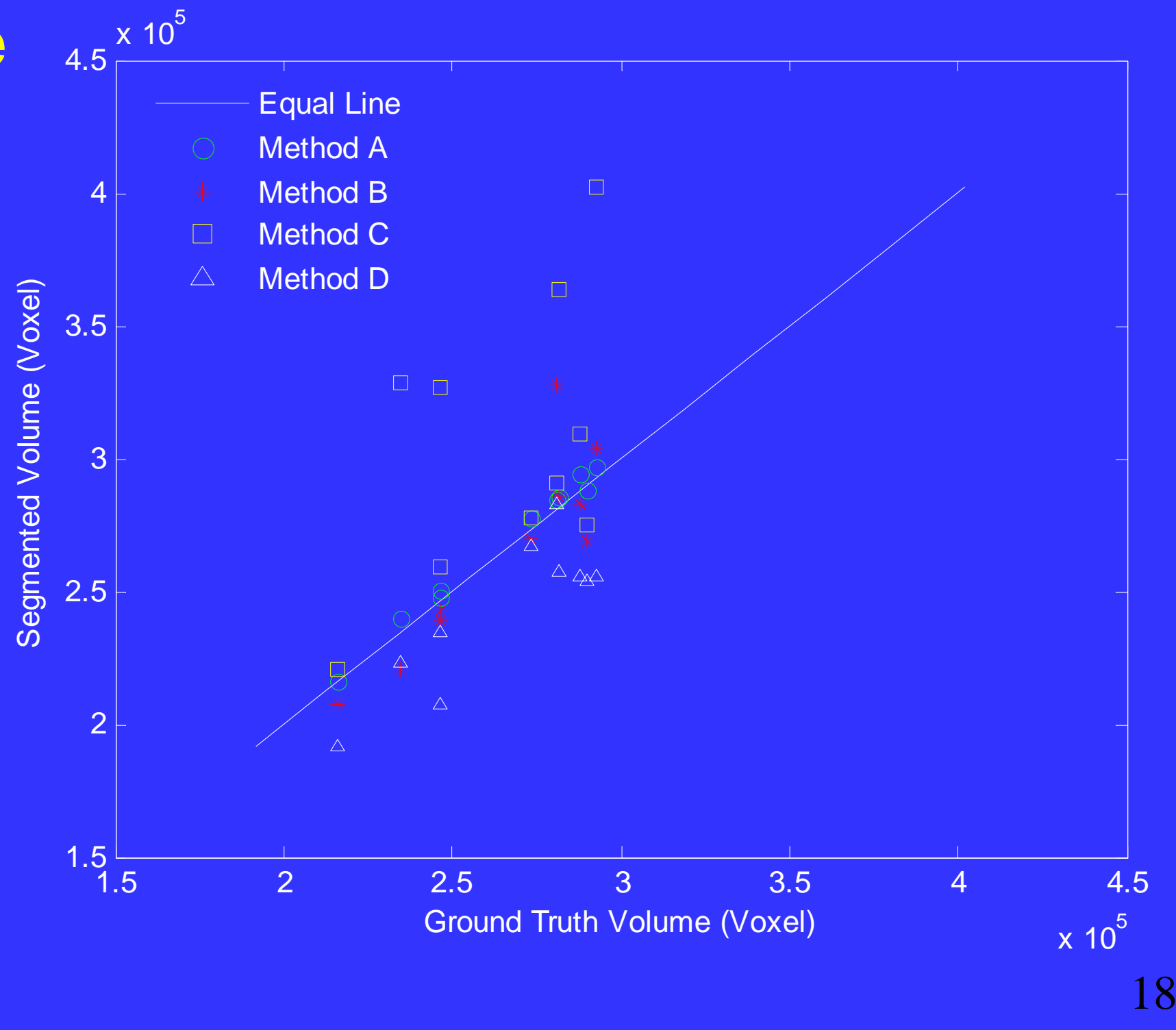




\section{Results}

\section{WM volume}

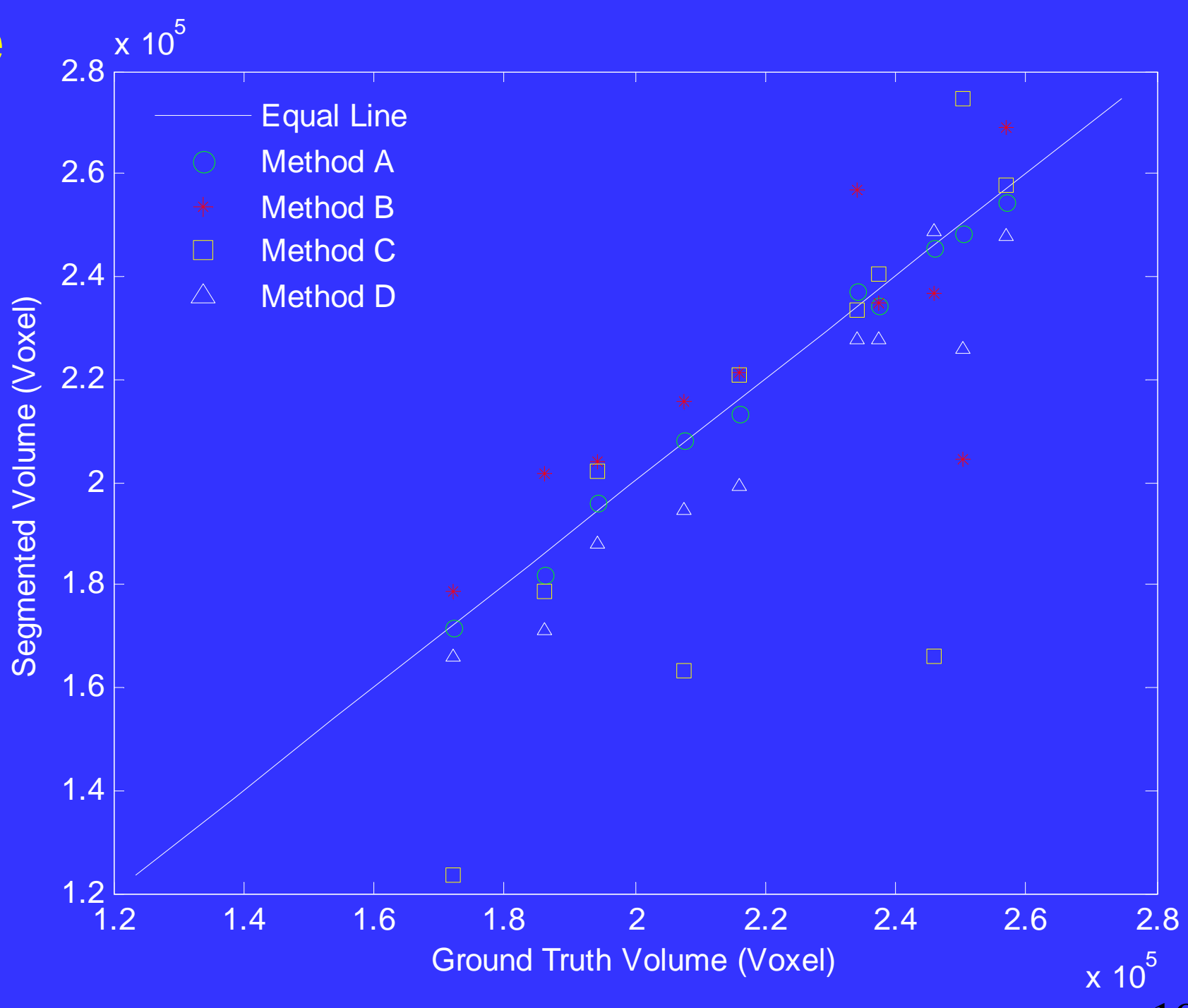




\section{Results}

\section{CSF volume}

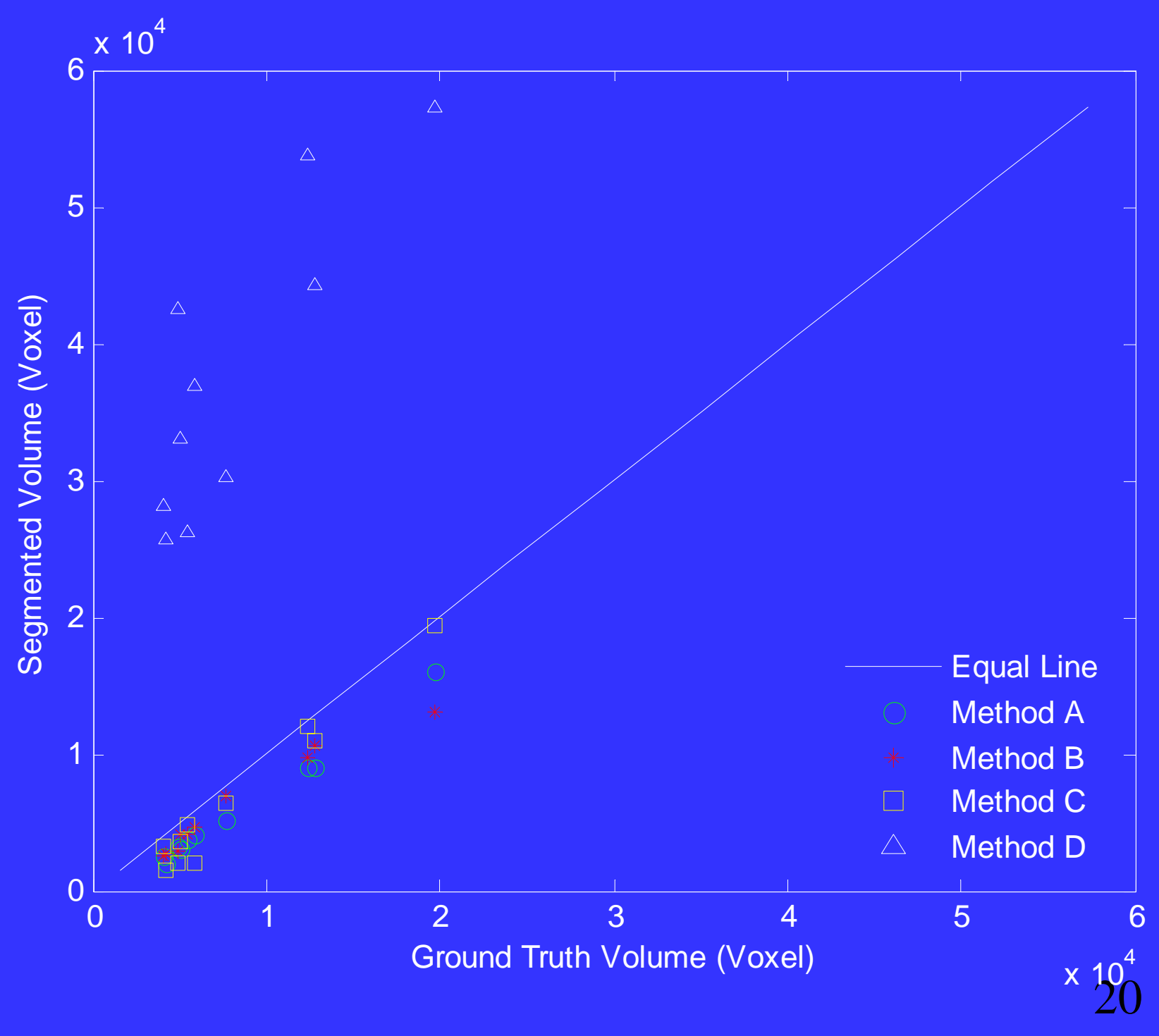




\section{Results}

\section{Accuracy Evaluation: True Positive}
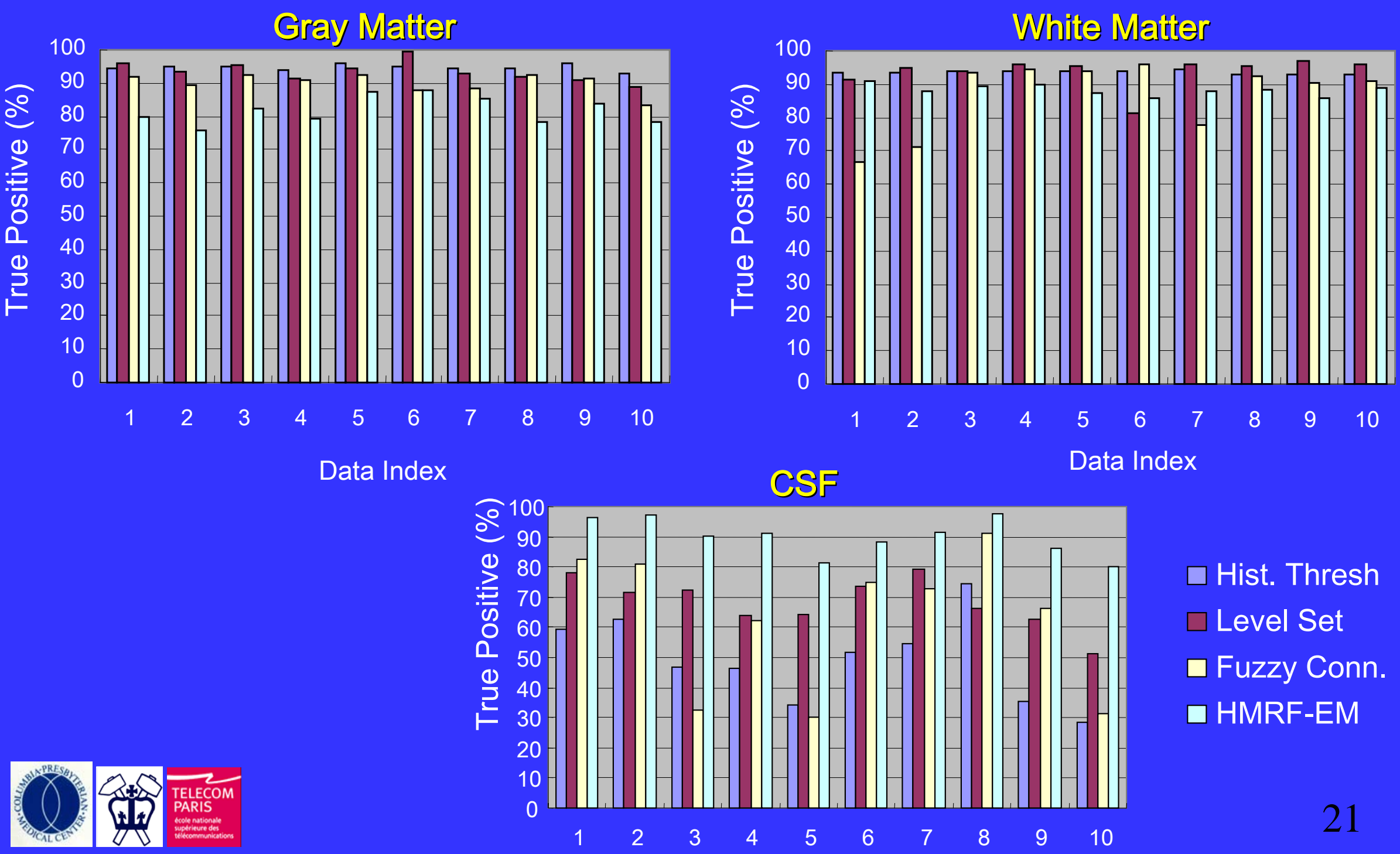

$\square$ Hist. Thresh

$\square$ Level Set

$\square$ Fuzzy Conn.

$\square$ HMRF-EM 


\section{Results}

\section{Accuracy Evaluation: False Positives}

Gray Matter

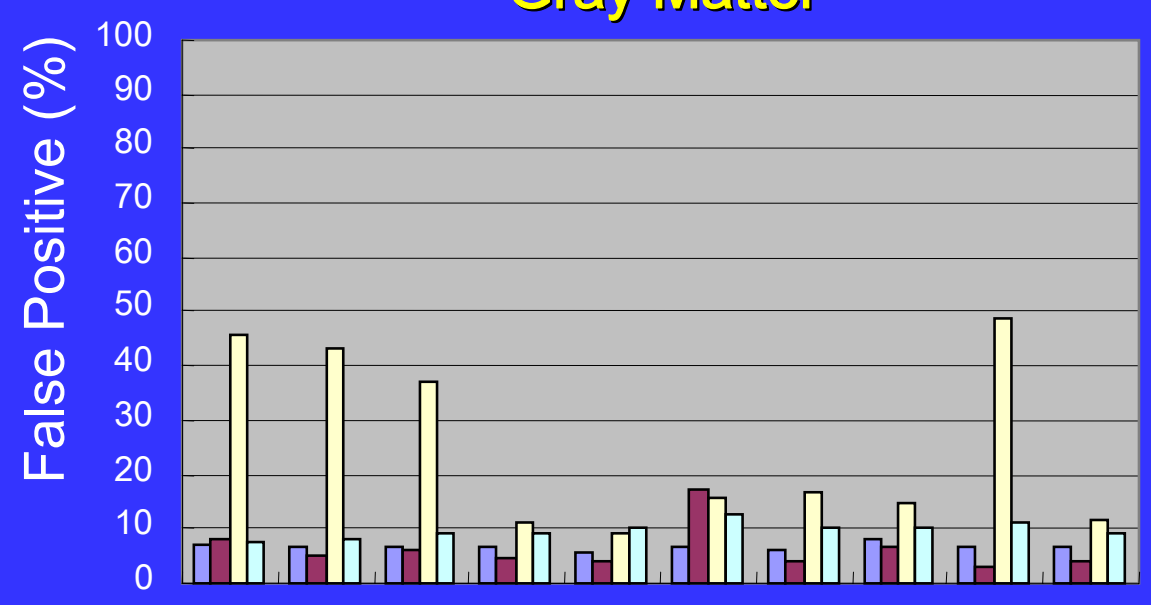

$\begin{array}{llllllllll}1 & 2 & 3 & 4 & 5 & 6 & 7 & 8 & 9 & 10\end{array}$

Data Index

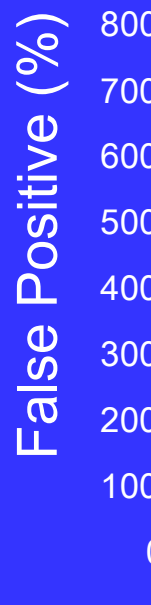

White Matter

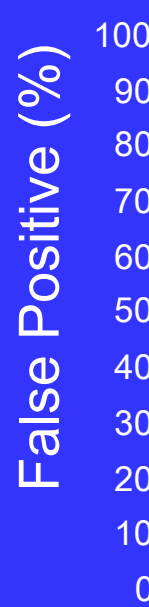

and
CSF

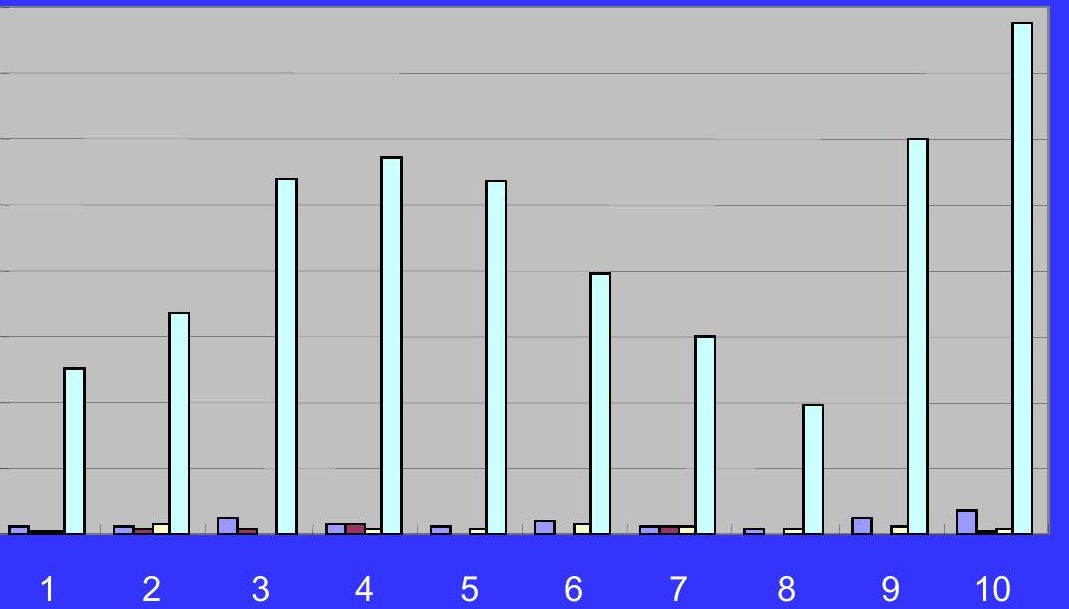

$\square$ Hist. Thresh.

$\square$ Level Set

$\square$ Fuzzy Conn.

$\square$ HMRF-EM 


\section{Results}

- Analysis of variance: ANOVA

- Inter-method variance / Intra-method variance of the TI index.

- Statistical difference between methods confirmed for $p<0.005$.
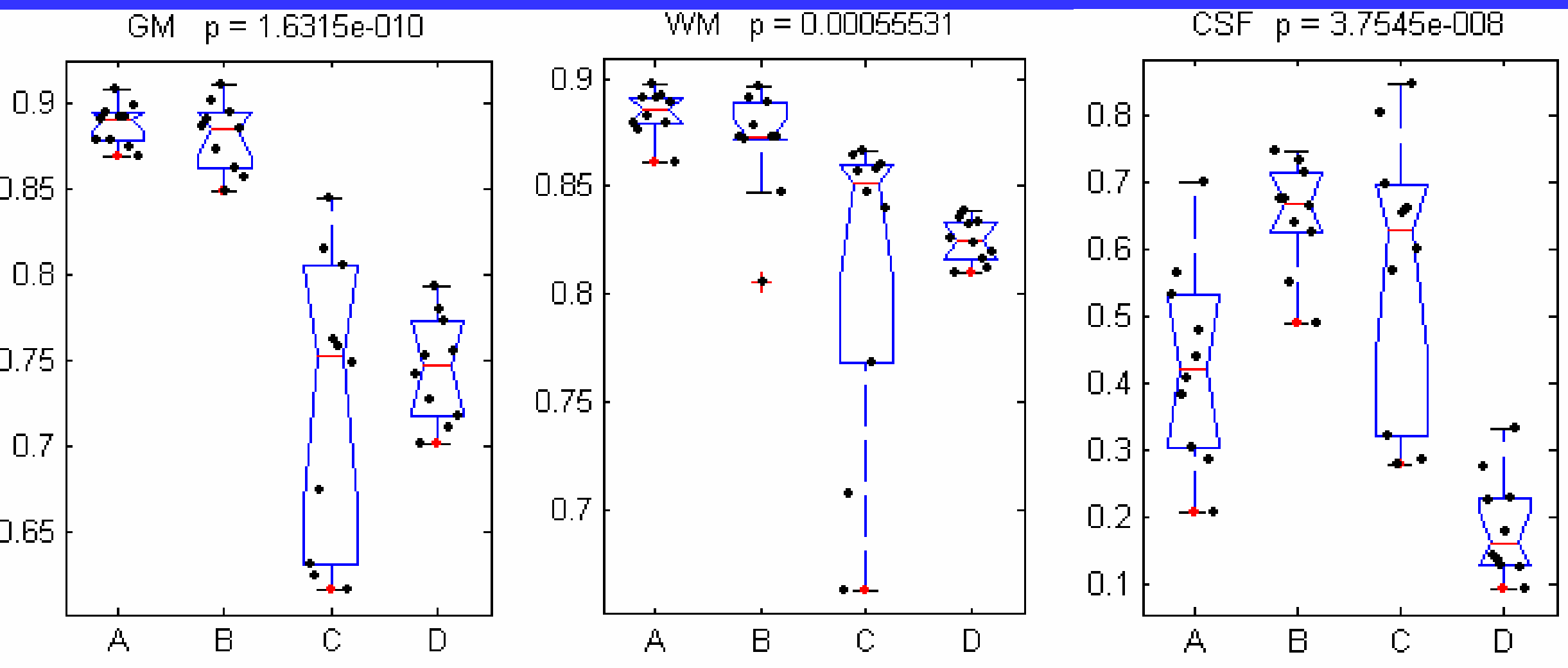


\section{Discussion}

- Segmentation of WM \& GM

- All methods reported high TI values.

- Superior performance of methods A and B.

- Segmentation of CSF

- Superiority of methods B and C (cf. TI values).

$\square$ A. Hist. Thresh.

$\square$ B. Level Set

- Highest variance for method C.

口C. Fuzzy Conn.

DD. HMRF-EM

- Significant under segmentation of CSF (i.e. high FN errors) due to very low resolution at the ventricle borders.

- Difference between methods for sulcal CSF:

- Different handling of partial volume effects

- Manual labeling eliminates sulcal CSF. Arbitrary choice and no ground truth available for these voxels.

- Manual labeling of the ventricles and sulcal CSF can vary up to $15 \%$ between experts as reported in the literature. 


\section{Conclusions}

- Four different methods were compared using clinical data.

$\square$ A. Hist. Thresh.

$\square$ B. Level Set

C. Fuzzy Conn.

- Statistical difference of methods was assessed.

$\square$ D. HMRF-EM

- Difference of performance focused on the extraction of CSF structures.

- Method A and B have strong correlations with manual tracing.

- Method C tends to over segment the GM structure in several cases.

- Method D tends to over segment the CSF structures.

- Combining all results, the level set three-dimensional deformable model (Method B) provides the best performance for high accuracy and low variance of performance index. 


\section{References}

1. E. D. Angelini, T. Song, B. D. Mensh, A. Laine, "Multi-phase threedimensional level set segmentation of brain MRI," MICCAI (Medical Image Computing and Computer-Assisted Intervention) International Conference 2004, Saint-Malo, France, September 26-30, 2004.

2. E. D. Angelini, T. Song, B. D. Mensh, A. Laine, "Segmentation and quantitative evaluation of brain MRI data with a multi-phase threedimensional implicit deformable model,"

SPIE International Symposium, Medical I maging 2004, San Diego, CA USA, Vol. 5370, pp. 526-537, 2004.

3. Heffner Biomedical I maging Lab http://hbil.bme.columbia.edu 